\title{
Evaluation of road safety measures efficiency in conditions of uncertainty
}

\author{
Vladimir Chernyshev ${ }^{1}$, Vladimir Klyavin ${ }^{2 *}$, and Vladimir Suvorov ${ }^{2}$ \\ ${ }^{1}$ Orel State University, 302026 Orel, Russia \\ ${ }^{2}$ Lipetsk State Technical University, 398600 Lipetsk, Russia
}

\begin{abstract}
Scientific approaches and methods are considered of the socialeconomic evaluation of the efficiency of a set of measures on improving road safety in conditions of uncertainty are considered. The risk levels for the adopted social-economic indicators were determined with the help of expert evaluation presented as a fuzzy number. In order to make a decision on a set of measures to be implemented, the scenario method, fuzzy linear programming, production rules were used. An integral indicator of the social-economic efficiency of measures is proposed as an objective means of assessment.
\end{abstract}

\section{Introduction}

Making managerial decisions on improving road safety requires a preliminary socialeconomic evaluation of the effectiveness of planned activities. In order to solve this problem, the method of the comparative analysis of changes in the number of road accidents, the number of fatalities, injuries and the amount of social and material damage for a certain period before and after the implementation of the activities is used and the average reduction in the number of accidents resulting from the activities is calculated [1], which does not provide the most effective choice of measures on improving road safety. Moreover, the managerial decision has to be made in an uncertainty, when it becomes necessary to take into account the impact of various risks. When developing managerial decisions, the scenario method is widely used to evaluate the most likely course of events and the possible consequences of decisions mad [2]. The scenario approach to analyzing the success of measures implemented to reduce the probability of road accidents involves the calculation of three options for development: pessimistic, optimistic and anticipated. The scenario approach proposed by Abt, Foster, and Rea [3] is the most suitable for the considered problem.

The peculiarity of this method is that, first of all, the development of scenarios refers to the analysis of a possible rather than a probable future, which implies the possibility of correction. The method involves selecting only those variables that are directly related to the development of the analyzed system, as well as the selection among a set of possible scenarios that are most suitable for subsequent analysis. The social-economic efficiency of measures and the discounted costs are taken as indicators of evaluation. It is most appropriate

* Corresponding author: vllk@list.ru 
to adopt the budget allocated for the implementation of the activities as the parameter which determines the restriction.

\section{Evaluating the efficiency of a set of measures in conditions of uncertainty}

Given the uncertainty associated with the market economy and the subjective perception of the expert, it is necessary to determine the risks of changing the adopted indicators. In order to do this, it is required to introduce linguistic variables to assess the risks of social-economic indicators: $x_{t}-$ evaluation of risk criteria. The term-set is the number of risk gradations $T$ $\{$ high, medium, low $\}$.

In this case, the values of the indicators will be represented as a fuzzy number. The fuzzy number $\mu_{A}(x)$ is a convex fuzzy set $A$ determined on the set of real numbers $R$ with a unimodal membership function (Figure 1). The fuzzy number is represented in the simplest form (triangle). It can be represented by a more complex dependence, which will increase the complexity of calculations, but will not influence the accuracy of the result significantly.

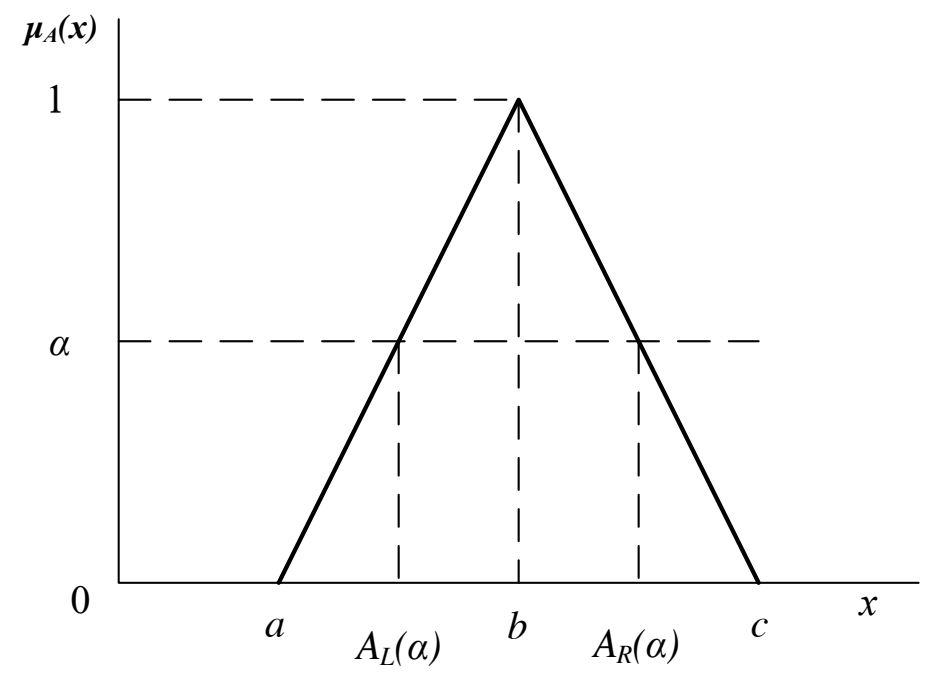

Fig. 1. Graph of term-set element membership functions

The left boundary of the membership function $a$ determines the pessimistic scenario $C_{p}$ for social-economic efficiency and budget, the right boundary $c$ is the optimistic scenario $C_{o}$. For the discounted costs, the boundaries change places. At point $b$, the expected value of $C_{e}$ is observed.

$\alpha$ is the specified minimum level of membership degrees which determine the subjective evaluation of the degree of confidence of the decision-maker in the possibility of risk events occurrence.

The $\alpha$-level set of the fuzzy set $A$ denoted as $A_{\alpha}$ is that part of its carrier for the elements of which the degree of membership in the given fuzzy set is not less than $\alpha$.

The $\alpha$-level set $(0 \leq \alpha \leq 1)$ is the interval $\left[A_{L}(\alpha), A_{R}(\alpha)\right]$. The left boundary of the interval $A_{L}(\alpha)=a+(b-a) \alpha$ is the distance between the left boundary and 0 . The right boundary of the interval $A_{R}(\alpha)=c-(c-b) \alpha$ is the distance between the right boundary and 0 .

There are $P$ indicators to be evaluated and analyzed by the risk degree according to $n$ criteria (Figure 2). To evaluate risks and weights, $m$ experts are involved. The criterion $i$ and its weight are evaluated by the expert $j$ in a qualitative form represented as a fuzzy number [4]. The method for processing expert evaluations is given in $[5,6]$. 
For a linear evaluation of the distance between fuzzy finite sets, the generalized Hamming distance is used. It is determined for fuzzy sets A and B given on the same $x$ [4]:

$$
d(A, B)=\left|\mu_{A}\left(x_{1}\right)-\mu_{B}\left(x_{1}\right)\right|+\ldots+\left|\mu_{A}\left(x_{n}\right)-\mu_{B}\left(x_{n}\right)\right|
$$

In order to determine the distance between the fuzzy number and the fuzzy sets constituting the totality of terms, it is necessary to convert fuzzy numbers into linguistic variables. The term for which distance is minimal determines the linguistic risk assessment for the indicator used to correct the membership function that determines the existence area of the social-economic indicator. The higher the risk level of the negative change in the parameter, the greater the value of $\mu_{A}(x)$. For $\mu_{A}(x) \Rightarrow 1$, the value of $C_{e}$ should be shifted to the value of $C_{p}$.

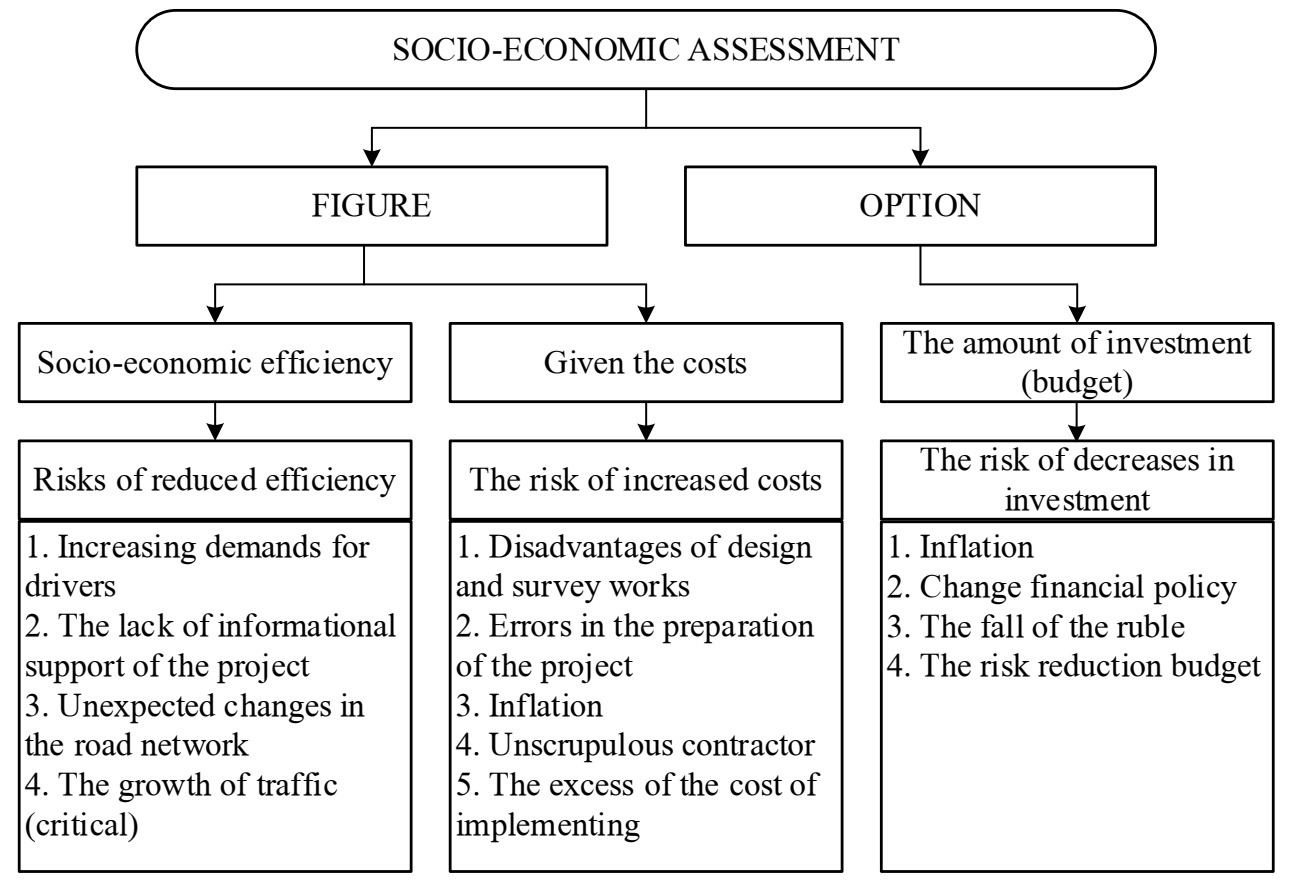

Fig. 2. Indicators and parameters of economic evaluation and risk criteria

The offset value Cec is calculated via linear interpolation:

- for efficiency and budget according to the formula

$$
C_{e c}=C_{e}-(\tau-1) \frac{C_{e}-C_{p}}{T-1} ;
$$

- for expenses (costs) according to the formula

$$
C_{e c}=C_{e}-(\tau-1) \frac{C_{p}-C_{e}}{T-1} ; \sum_{i=1}^{m} s_{i} x_{i} \leq u,
$$

where $\tau$ - linguistic risk evaluation ( 0 - "low", 1 - “medium", 2 - "high");

$T$ - number of risk gradations $(T=3)$.

In order to make a managerial decision, it is necessary to solve a task of fuzzy linear programming (Figure 3) [7-9]: 


$$
C_{a}(X)=\sum_{i=1}^{m} e_{i} x_{i} \sim \triangleright \max
$$

if

$$
\sum_{i=1}^{m} s_{i} x_{i} \leq u,
$$

where $C_{\alpha}(X)$ - fuzzy effect from implementing a set of measures on the level $\alpha\left(x_{i}=0\right.$, if the measure is not implemented, $x_{i}=1$, if the measure is implemented); $m$ - number of objects where it is proposed to implement measures to improve road safety; $e_{i}$ - fuzzy socialeconomic efficiency of the implementation of measures at the $i$ site; $s_{i}$ - fuzzy discounted costs for the implementation of measures at the $i$ site; $u$ - fuzzy budget allocated for the implementation of all measures.

The operation " $\leq$ " is understood as fuzzy. The symbol $\sim \triangleright$ means that among all vectors $X$ it is necessary to find such a vector $X^{0}$ for which $C_{\alpha}\left(X^{0}\right) \geq C_{\alpha}(X)$ in accordance with the fuzzy operation "greater than or equal to the level $\alpha$ "

The data on the traffic organization and accident rates in Gagarin Street, which is one of the streets in Lipetsk with high accident rates, was used as an example [14, 15]. 11 "blackspot" sites were identified for which a set of necessary measures on improving road safety was determined from the following list: bus bay (sites 1, 3, 5, 7-11), controlled crossing (sites 2, 4, 6, 7, 9, 10), safety island (sites 4, 9, 10), pedestrian guard rails (sites 2-10) (see Table 1). The discounted costs for the implementation of measures are recommended by the experts of the Department of roads and transport of the Lipetsk region, the damage from casualties was calculated according to the research data of HSE [10], vehicles' mechanical damages were calculated grounding on the average Obligatory Motor Insurance payments. The indicator "social-economic effect of the implementation of measures" was calculated as prevented damage depending on the development scenario: $100 \%$ - optimistic, $75 \%$ expected, $50 \%$ - pessimistic.

\begin{tabular}{|c|c|c|c|c|c|c|c|}
\hline \multirow[t]{2}{*}{$\begin{array}{c}\text { Site } \\
\text { number }\end{array}$} & \multicolumn{3}{|c|}{$\begin{array}{c}\text { Discounted costs for implementation } \\
\text { of measures, mln roubles. }\end{array}$} & \multirow{2}{*}{$\begin{array}{c}\text { Damage, } \\
\text { mln } \\
\text { roubles. }\end{array}$} & \multicolumn{3}{|c|}{$\begin{array}{c}\text { Social-economic effect of } \\
\text { implementation of measures, } \mathrm{mln} \\
\text { roubles. }\end{array}$} \\
\hline & Optimistic & Expected & Pessimistic & & Optimistic & Expected & Pessimistic \\
\hline 1 & 0.6 & 0.8 & 1 & 2,48506 & 2.48506 & 1.863795 & 1.24253 \\
\hline 2 & 1.2 & 1.8 & 2.4 & 10,21593 & 10.21593 & 7.661945 & 5.107963 \\
\hline 3 & 0.8 & 1,1 & 1.4 & 1,679309 & 1.679309 & 1.259482 & 0.839655 \\
\hline 4 & 1.12 & 1.68 & 2.24 & 1,234836 & 1.234836 & 0.926127 & 0.617418 \\
\hline 5 & 0.8 & 1.1 & 1.4 & 0,694062 & 0.694062 & 0.520546 & 0.347031 \\
\hline 6 & 1.3 & 1.95 & 2.6 & 1,193238 & 1.193238 & 0.894928 & 0.596619 \\
\hline 7 & 2 & 2.9 & 3.8 & 3,184532 & 3.184532 & 2.388399 & 1.592266 \\
\hline 8 & 0.7 & 0.95 & 1.2 & 2,498165 & 2.498165 & 1.873624 & 1.249082 \\
\hline 9 & 2.82 & 4.03 & 5.24 & 1,471319 & 1.471319 & 1.103489 & 0.73566 \\
\hline 10 & 2.52 & 3.58 & 4.64 & 9,160587 & 9.160587 & 6.87044 & 4.580294 \\
\hline 11 & 0.7 & 0.95 & 1.2 & 10,59572 & 10.59572 & 7.946789 & 5.297859 \\
\hline
\end{tabular}

Table 1. Original data for solving the problem of fuzzy linear programming. 


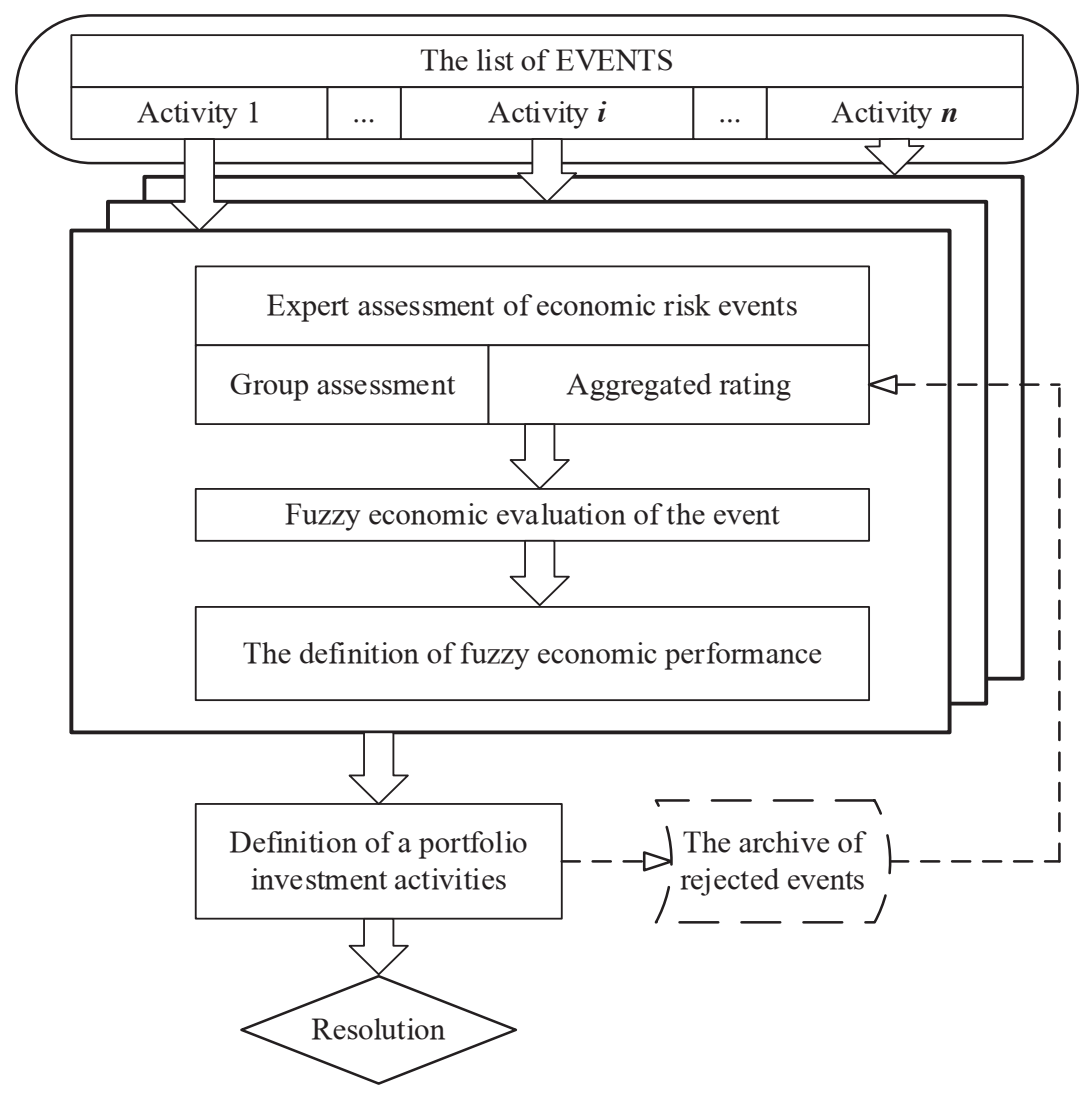

Fig. 3. Algorithm of solving the problem of managerial decision-making.

The problem of fuzzy linear programming was solved via the Microsoft Excel Solution Search add-in. For this purpose, a transition was made from a "fuzzy" problem formulation to a clear one by means of "if-then" production rules [11] which are determined by the person making the decision. If the measure is not going to be implemented completely, then the requirement of integer solution can be replaced by a milder requirement $0 \leq x_{i} \leq 1$.

Then, the rule "If: the risk of reducing the effect is high - Then: use the right boundary of the fuzzy set" is used as an example. A problem solution for the level $\alpha=0.9$ is given (Fig. $4)$.

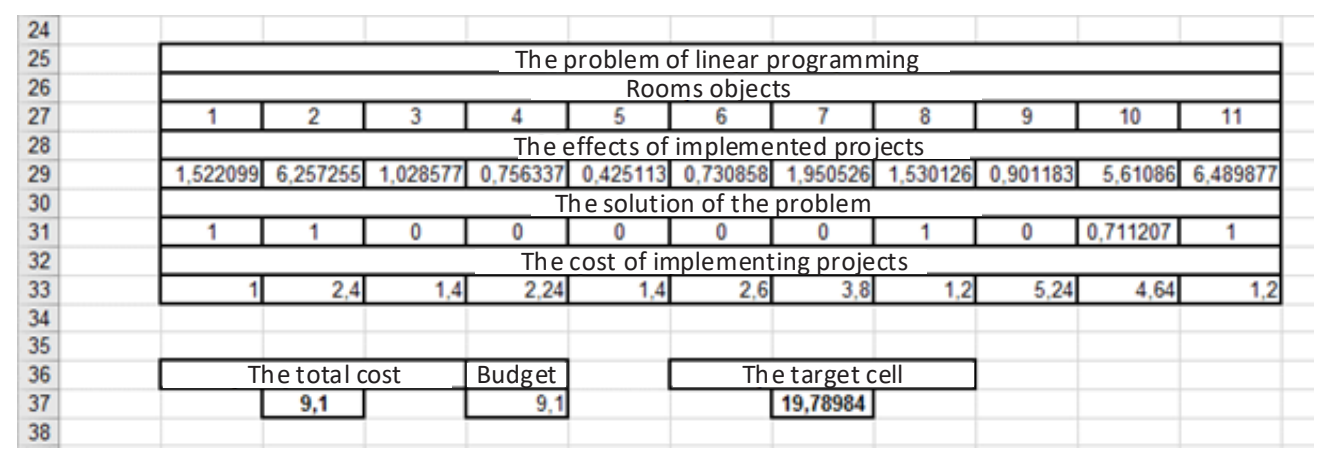

Fig. 4. Solving the problem via Microsoft Excel Solution Search add-in 
The analysis of the calculation results showed that at the costs corresponding to the expected value of the budget (9.1 million rubles) the implementation of the whole complex of measures is possible at sites $1,2,8,11$. At site 10 , the implementation of the complex of measures will be implemented by $71.1 \%$.

In order to objectively evaluate the decision making, it is recommended to use the integral indicator - the value of the social-economic effect $k_{\alpha}$, obtained per 1 rouble of discounted costs:

$$
I_{p}=E_{a} / S_{d i s}
$$

where $E_{\alpha}$ - social-economic efficiency; $S_{d i s}$ - discounted costs for the implementation of measures at the level of $\alpha$, roubles.

The integral index is calculated for each level $\alpha$. The data obtained are presented for further analysis in the form of a graph (Figure 5) which shows the areas corresponding to the ranking of the degree of the decision-maker's confidence or awareness of economic risks in the implementation of measures: $0<\alpha \leq 0.2$ - "not sure"; $0.2<\alpha \leq 0.5$ - "weakly sure"; 0,5 $<\alpha \leq 0.8$ - "not sure enough"; $0.8<\alpha \leq 1$ - "sure."
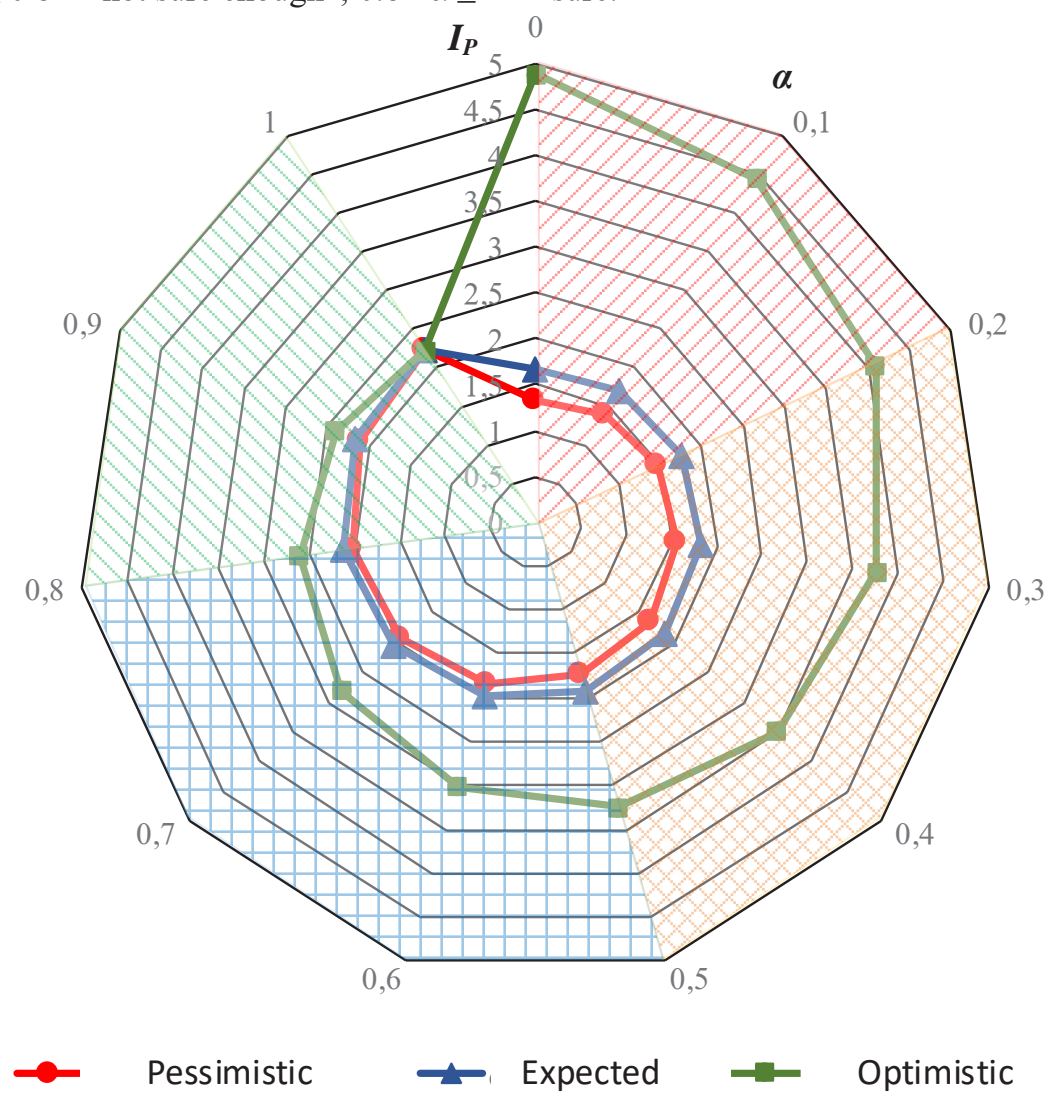

Fig. 5. Dependence of the integral indicator on the $\alpha$-level

Depending on the degree of confidence, the "blurring" of the values of the integral indicator $I_{P}$ may vary widely. So, for example, at the level $\alpha=0.1$, the values lie in the range from 1.41 to 4.46 , and at the level $\alpha=0.9$ they are in the range from 2.12 to 2.40 . The creation of a database accumulating the results of implemented measures will significantly increase the significance and accuracy of the social-economic evaluation. 


\section{Conclusion}

Implementation of the project "Road safety in 10 countries" (RS10) in Russia showed the effectiveness of measures to improve road safety $[12,13]$. The developed scientific and applied toolkit for the social-economic evaluation and selection of the most effective set of measures to improve road safety in conditions of uncertainty is consistent with the methodology of the expert system "Road safety" [14,15], but can be used independently. The application of the obtained scientific results provides an opportunity for a qualitative evaluation of road safety measures whose main goal is to save human lives and to reduce road traffic injuries.

\section{References}

1. I.N. Pugachev, Road organization and safety: Manual for universities. Moscow, Academia (2009)

2. B.G. Litvak, Development of managerial decisions: Manual for universities. Moscow, Delo (2000)

3. C.C. Abt, R. Foster, R.H. Rea, A scenario generating methodology. In: Manual of scientific and technical forecasting. Moscow, Progress (1977)

4. L. Kofman, Introduction to the fuzzy sets theory: Translated from French. Moscow, Radio i Svyaz (1982)

5. V. Korchagin, A. Pogodaev, V. Kliavin, V. Suvorov A method of complex evaluation of the road network safety level, Vestnik MADI, 2 (45), p. 88-94 (2016)

6. V. Korchagin, A. Pogodaev, V. Kliavin, V. Suvorov, A method of objective evaluation of the road safety level, Science and technology in road engineering, 1, p. 10-12 (2017)

7. B. Liu, Theory and Practice of Uncertain Programming, 2nd ed., http://orsc.edu.cn/liu/up.pdf.

8. A. Piegat, Fuzzy modeling and control. Heidelberg, Physica-Verlag (2001)

9. S. Young, System organization control, Translation from English edited by S.P. Nikanorov, S.A. Batasov. Moscow, Sovetskoye radio (1972)

10. E.M. Reshetova, Institutional factors of improving road safety, Analitical Vestnik of the Federation Council of the Federal Assembly of Russia, 33 (632), p. $46-58$ (2016)

11. J. Giarratano, and G. Riley, Expert Systems: Principles and Programming. 4th Edition: (Hardcover), ISBN: 0534384471 (2004)

12. S. Ma, A.A. Hyder, N. Tran, F. Zambon, V.E. Klyavin, K.W. Hatcher, A. B. Petruhin, E.S. Slyunkina, F. Zambon Seat belt and child seat use in Lipetskaya Oblast, Traffic Injury Prevention, 13 (1). pp. 76-81 (2012)

13. V.E. Kliavin, S. Gupta, N. Paichadze, E. Gritsenko, E. Yurasova, A.A. Hyder, Evaluation of the five-year Bloomberg Philanthropies Global Road Safety Program in the Russian Federation, Public health, 144, pp. 5-14 (2017)

14. V. Korchagin, S. Lyapin, V. Kliavin, V. Sitnikov, An expert system of road safety monitoring and control, Vestnik of Lipetsk State Technical University, 3 (29), p. 33-38 (in Russian) (2016)

15. V. Korchagin, A. Pogodaev, V. Kliavin, V. Sitnikov, Scientific Basis of the Expert System of Road Safety, Transportation Research Procedia, 20, pp. 321 - 325 (2017) 